\title{
Saúde bucal na escola: revisão integrativa
}

\section{Oral health at school: integrative review}

Salud bucal en la escuela: revisión integrativa

Received: 08/02/2021 | Reviewed: 08/06/2021 | Accept: 08/10/2021 | Published: 08/14/2021

\author{
Anny Vanessa Cavalcante Costa Silva \\ ORCID: https://orcid.org/0000-0001-9690-938X \\ Centro Universitário Mario Pontes Jucá, Brasil \\ E-mail: an.vanessa@hotmail.com \\ Bráulio Sía de Queiroz Junior \\ ORCID: https://orcid.org/0000-0001-5943-766X \\ Centro Universitário Mario Pontes Jucá, Brasil \\ E-mail: siajunior@hotmail.com \\ Ana Maria Guerra Costa \\ ORCID: https://orcid.org/0000-0002-6564-4718 \\ Centro Universitário Mario Pontes Jucá, Brasil \\ E-mail: anaguerracosta@yahoo.com.br
}

\begin{abstract}
Resumo
O estudo demonstra atualmente os esforços da odontologia sendo analisada através da prevenção das doenças bucais, e demais patologias, destacando as necessidades de ensinar e a motivação dos melhores hábitos de higiene bucal da criança, adotando a educação em saúde voltada na aprendizagem da melhoria da qualidade de higiene bucal no dia a dia. O objetivo da pesquisa foi relatar programas de educação de saúde bucal na escola e a motivação para criança em relação à saúde bucal em escolas. Esta revisão sistemática relata os cuidados com a higiene oral e são o foco da atuação da odontologia atualmente. Além de trabalhar com a prevenção em todos os pacientes, os pequenos devem compreender desde cedo a importância de seguir os cuidados com a saúde_bucal e como isto afeta seu desenvolvimento até a vida adulta.
\end{abstract}

Palavras-chaves: Educação em saúde bucal; Saúde bucal; Doenças de boca; Higiene bucal; Educação em odontologia.

\begin{abstract}
The study currently demonstrates the efforts of dentistry being analyzed through the prevention of oral diseases, in addition to pathologies, seeing highlighting the needs of teaching and the motivation of the best oral hygiene habits of children, thus adopting health education aimed at learning how to improve the quality of daily habits. The objective of the research was to report on oral health education programs at school and the motivation for children in relation to oral health in schools. So, the systematic review reports care is the focus of dentistry today. In addition to working with prevention in all patients, young people must understand from an early age the importance of following oral health care, and how it affects their development and adult life.
\end{abstract}

Keywords: Oral health education; Oral health; Mouth diseases; Oral hygiene; Dentistry education.

\section{Resumen}

El estudio demuestra actualmente los esfuerzos de la odontología siendo analizados a través de la prevención de enfermedades bucales y otras patologías, destacando las necesidades de enseñar y la motivación de los mejores hábitos de higiene bucal del niño, adoptando una educación para la salud enfocada en aprender a mejorar la calidad de la higiene bucal. en todos los días de la vida. El objetivo de la investigación fue informar sobre los programas de educación en salud bucal en la escuela y la motivación del niño en relación con la salud bucal en las escuelas. Esta revisión sistemática informa sobre el cuidado de la higiene bucal y actualmente es el foco de la odontología. Además de trabajar con la prevención en todos los pacientes, los niños deben comprender desde una edad temprana la importancia de seguir el cuidado de la salud bucal y cómo esto afecta su desarrollo hasta la edad adulta.

Palabras chave: Educación en salud bucal; Salud bucal; Enfermedades de la boca; Higiene bucal; Educación en odontología.

\section{Introdução}

O trajeto da saúde bucal no Brasil foi caracterizado por uma assistência odontológica pública deficiente e grande limitação do acesso da população, causando superlotação das Unidades Básicas de Saúde (UBS). Diante do quadro de problemas que a saúde bucal enfrentava se fez indispensável a elaboração de uma política nacional de saúde bucal, a qual foi desenvolvida e incentivada pelo Governo Federal. Em março de 2004, foi instaurado o Programa Brasil Sorridente, que possui normas e 
diretrizes que visam à ampliação e à qualificação do atendimento em saúde bucal na atenção básica, proporcionando à população uma realização de atividades de promoção e prevenção à saúde, possibilitando acessibilidade para todos os usuários do Sistema Público de Saúde (Bulgareli et al., 2014).

A OMS (Organização Mundial de Saúde), no documento reconhece a relação que existe entre educação e saúde; a partir disto, julga que se pode empregar este conhecimento para ajudar a estabelecer escolas que melhorem a educação e aumentem o potencial de aprendizagem, ao mesmo tempo que melhoram a saúde, pois a boa saúde apoia um aprendizado proveitoso e viceversa. Afirma-se que a escola tem grande influência sobre a saúde dos jovens. Daí o desenvolvimento do conceito de Escolas Promotoras da Saúde ou Escolas Saudáveis, que têm como meta genérica atingir estilos de vida saudáveis para a população total da escola por meio do desenvolvimento de ambientes que apoiem e conduzam à promoção da saúde (Leite, 2013).

A cárie dentaria e a doença periodontal são os males que mais afetam a cavidade oral. A educação e a informação sobre os cuidados com a mesma, tem sido ressaltadas por diversos pesquisadores, que destacam a relevância de programas odontológicos educativos. Através dos cuidados necessários de higiene bucal, o ambiente escolar torna-se o local mais indicado para construção desses, entretanto, instituir esses hábitos no cotidiano de forma rotineira, é o maior desafio para as instituições de ensino, visto que isso vai além dos muros que cercam a escola, ultrapassando apenas as ferramentas pedagógicas (Antonio et al., 2015).

A motivação e a educação em saúde, são poderosas ferramentas quando empregadas de maneira agradável, atrativa e eficaz para transmissão de informações, sendo este o objetivo da promoção de saúde nas escolas. Nesse sentido, a literatura enfatiza que a escola é um ambiente propício para a aplicação de programas de educação em saúde por estar inserida em todas as dimensões do aprendizado. Portanto a pergunta para nortear a pesquisa no campo da educação em saúde bucal foi: Qual a melhor forma da melhoria da qualidade da saúde bucal na escola?

\section{Metodologia}

O presente estudo trata-se de uma revisão sistemática, sendo um método de pesquisa concentrado no Âmbito da Prática Baseada em Evidências (PBE). Por ser um método, o mesmo consta das seguintes etapas: a primeira é a identificação do tema, e as seguintes são respectivamente: estabelecimento de critérios para inclusão e exclusão de estudos; definição das informações a serem extraídas dos estudos selecionados; avaliação dos estudos incluídos na revisão integrativa; interpretação dos resultados e apresentação da revisão. O método de saúde bucal na escola.

Para atingir o proposto estudo, realizou-se a busca e classificação de dados informativos na análise crítica dos artigos das bases eletrônica e também de livros que abordam sobre o tema onde foram utilizados: SCIELO, LILACS e MEDLINE, no período de 2011 à 2021, onde fica o uso de artigos na íntegra em português e espanhol.

Critérios de inclusão estabelecidos para esta pesquisa foram publicações na íntegra com acesso livre; com a temática relevante para atingir objetivos propostos. Adicionalmente, ressalta-se que foram consultadas literaturas presentes em arquivo pessoal e literaturas clássicas. Os critérios de exclusão são bibliografias que não correspondem com a temática proposta.

Todos os artigos identificados foram lidos na íntegra e caracterizados quanto aos autores e quanto à pesquisa ou estudo publicado, ano de publicação, base indexada e os resultados obtidos. Os resultados e conclusões serão apresentados a seguir de forma descritiva.

\section{Resultados}

Serão critérios de inclusão artigos na língua portuguesa, publicados entre 2011 a 2021, nos idiomas português e espanhol. Serão excluídos artigos incompletos com títulos que não estavam diretamente relacionados ao tema da pesquisa e/ou 
que não completavam informações relevantes relacionados ao objeto de estudo; também serão eliminados da amostra final os artigos em duplicidade.

Os artigos a serem compostos da amostra serão aqueles que tenham compatibilidade com a pesquisa e também como o estudo onde irão ser selecionados aqueles que tiverem em relação com o tema, visando os resumos e sendo eliminados os artigos que não correspondem à questão norteadora.

Haverá a definição das informações extraídas dos estudos onde serão organizados em tabelas de dados diante das categorias dos estudos, selecionados diante da tabulação de dados com títulos, ano de publicação, nome dos autores e desfecho desses artigos.

Os estudos incluídos na revisão sistemática foram analisados de forma crítica, tendo como foco do estudo, a questão norteadora para a elaboração do conteúdo, tendo como visto a síntese de conhecimento sobre o tema abordado.

Para a realização do estudo, as seguintes etapas foram estabelecidas: identificação do tema e da seleção da pergunta norteadora: estabelecimento de critérios de inclusão e exclusão de artigos (seleção da amostra); definição das informações a serem extraídas dos artigos selecionados; análise dos resultados; avaliação dos estudos incluídos na revisão integrativa; apresentação e discussão dos resultados e apresentação da revisão e dos resultados. Diante do exposto, os critérios de inclusão estabelecidos para esta pesquisa são artigos disponíveis integralmente on-line em português, publicados entre 2011 a 2021.

Tabela 1 - Estratégia de busca eletrônica.

\begin{tabular}{c|l|c|c}
\hline Banco de Dados & \multicolumn{1}{|c|}{ Palavras-chaves/Descritores } & Artigos encontrados & Artigos selecionados \\
\hline \multirow{2}{*}{ LILACS } & $\begin{array}{l}\text { 1- Educação em Saúde Bucal, Saúde } \\
\text { Bucal, Doenças de Boca; Higiene } \\
\text { Bucal; Educação em Odontologia. }\end{array}$ & 120 & 02 \\
\hline MEDLINE & $\begin{array}{l}\text { 2- Educação em Saúde Bucal, Saúde } \\
\text { Bucal, Doenças de Boca; Higiene } \\
\text { Bucal; Educação em Odontologia. }\end{array}$ & 45 & 02 \\
\hline SCIELO & $\begin{array}{l}\text { 3- Educação em Saúde Bucal, Saúde } \\
\text { Bucal, Doenças de Boca; Higiene } \\
\text { Bucal; Educação em Odontologia. }\end{array}$ & 26 & 04 \\
\hline
\end{tabular}

Fonte: Dados de Pesquisa (2021).

Para facilitar o entendimento dos leitores, segue abaixo a segunda tabela descrevendo as principais características dos artigos que foram utilizados no referencial teórico da pesquisa. Nesse contexto, a busca em base de dados procurou ser ampla e diversificada, envolvendo as bases eletrônicas, busca manual em periódicos. Na presente revisão integrativa, artigos encontrados, foram distribuídos da seguinte forma: LILACS, SCIELO, MEDLINE. Após a leitura dos títulos, descritores e resumos, foram incluídos 8 artigos para compor a amostra. E por fim, foi feita a interpretação dos resultados encontrados, previamente estabelecidos e estão representadas no Quadro 1.

\section{Discussão}

Todos os artigos identificados foram lidos na íntegra e caracterizados quanto aos autores e quanto à pesquisa ou estudo publicado, ano de publicação, base indexada e os resultados obtidos. Os resultados e conclusões serão apresentados a seguir de forma descritiva. A informação selecionada foi organizada no Quadro (2), por ordem de publicação (do mais antigo ao mais recente) onde constam dados relativos aos autores, ano de publicação, onde foram publicados o estudo e o objetivo geral nos estudos. 
Quadro 2 - Apresentação dos artigos selecionados com objetivos de pesquisa.

\begin{tabular}{|c|c|c|c|c|}
\hline ANO & AUTORES & TITULO & $\begin{array}{c}\text { BASE } \\
\text { INDEXADA }\end{array}$ & RESULTADOS E CONCLUSÕES \\
\hline 2011 & $\begin{array}{l}\text { VALARELLI; F, P; } \\
\text { FRANCO; VALARELLI, } \\
\text { F.P; FRANCO; R, M; } \\
\text { SAMPAIO; C, C; } \\
\text { MAUAD C; PASSOS; } \\
\text { L.L; VITOR; L, L, R, V; } \\
\text { MACHADO; M, A.A.M; } \\
\text { OLIVEIRA, T; M }\end{array}$ & $\begin{array}{l}\text { Importância dos } \\
\text { programas de } \\
\text { educação motivação } \\
\text { para saúde bucal em } \\
\text { escolas: relato de } \\
\text { experiência }\end{array}$ & LILACS & $\begin{array}{l}\text { Escola tem sido considerada um local adequado para o } \\
\text { desenvolvimento de programas de saúde, possibilitando } \\
\text { ações de promoção e prevenção em saúde bucal, pois o } \\
\text { período escolar é um momento da vida em que a criança } \\
\text { se encontra mais apta a agregar conhecimentos, agindo, } \\
\text { dessa forma, o professor, como modelador de } \\
\text { comportamentos na saúde da criança, pelo contato } \\
\text { diário durante longo período de tempo. }\end{array}$ \\
\hline 2012 & $\begin{array}{lcc}\text { CASTRO; } & \text { C, } & \text { O; } \\
\text { OLIVEIRA; } & \mathrm{K}, & \mathrm{S} ; \\
\text { CARVALHO; } & \mathrm{R}, & \mathrm{B} ; \\
\text { GARBIN, C, } & \mathrm{A}, & \mathrm{S} ; \\
\text { SANTOS, K.T } & & \end{array}$ & $\begin{array}{l}\text { Programas de educação e } \\
\text { prevenção em saúde } \\
\text { bucal nas escolas análise } \\
\text { crítica de publicações } \\
\text { nacionais. }\end{array}$ & SCIELO & $\begin{array}{l}\text { Na Odontologia, a educação em saúde pode esclarecer } \\
\text { a população sobre a importância dos hábitos de higiene } \\
\text { bucal com procedimentos simples e baratos e, ainda, } \\
\text { como eles influenciam a saúde e a vida cotidiana das } \\
\text { pessoas. }\end{array}$ \\
\hline 2012 & $\begin{array}{l}\text { SANTOS; K, T; } \\
\text { GARBIN.A; J; I; } \\
\text { GARBIN, C, A, S; }\end{array}$ & $\begin{array}{l}\text { Saúde Bucal nas } \\
\text { escolas: relato de } \\
\text { experiência }\end{array}$ & SCIELO & $\begin{array}{l}\text { A necessidade de aproximação entre acadêmicos e a } \\
\text { realidade vivenciada pela comunidade, bem como a de } \\
\text { educar buscando a transformação social, desde uma } \\
\text { tenra idade, levaram ao desenvolvimento do Projeto de } \\
\text { Extensão de Educação em Saúde }\end{array}$ \\
\hline 2016 & $\begin{array}{llr}\text { GARBIN, } & \text { C, A, } & \text { S; } \\
\text { SOARES; } & \text { G, } & \text { B; } \\
\text { MARTIN; } & \text { I, } & \text { M; } \\
\text { GARBIN; A, J, I; } & \text { ARCIERI; M, A; }\end{array}$ & $\begin{array}{l}\text { Saúde bucal na } \\
\text { escola: avaliação do } \\
\text { conhecimento dos } \\
\text { pais e da condição de } \\
\text { saúde bucal das } \\
\text { crianças }\end{array}$ & MEDLINE & $\begin{array}{l}\text { Educação em saúde é uma importante ferramenta para } \\
\text { levar ao indivíduo mais conhecimento na busca por } \\
\text { orientá-lo para mudanças de atitudes e desenvolvimento } \\
\text { de hábitos saudáveis. }\end{array}$ \\
\hline 2017 & $\begin{array}{l}\text { SOUSA; J, B; LIMA; } \\
\text { ELIDA MARIA } \\
\text { MARCOS; BENTO, A, } \\
\text { K, M; QUEIROZ; LUIZ, } \\
\text { G, S; SILVA; C, H, F. }\end{array}$ & $\begin{array}{l}\text { Saúde Bucal na } \\
\text { escola: Um estudo } \\
\text { sobre atividades de } \\
\text { educação em saúde } \\
\text { para estudantes. }\end{array}$ & SCIELO & $\begin{array}{l}\text { Apesar do tema educação em saúde bucal ainda ser um } \\
\text { desafio e existirem diversos programas que versam } \\
\text { sobre os seus cuidados, ainda existe uma grande } \\
\text { escassez no repasse de informações que levem as } \\
\text { pessoas à conscientização da importância dos cuidados } \\
\text { com a saúde bucal. }\end{array}$ \\
\hline 2017 & $\begin{array}{lr}\text { PEREIRA; } & \text { G; } \\
\text { CARNEIRO; } & \text { S.V; } \\
\text { MARTINS; } & \text { L.F.B; } \\
\text { BENTO; A, K, M; LEITE; } \\
\text { A, C, R, M; SILVA; } \\
\text { C.H.F }\end{array}$ & $\begin{array}{l}\text { A promoção da saúde } \\
\text { bucal no contexto } \\
\text { escolar: uma revisão } \\
\text { integrativa }\end{array}$ & LILACS & $\begin{array}{l}\text { O ambiente escolar tem grande influência sobre a saúde } \\
\text { dos jovens, tornando-se um local propício para o } \\
\text { desenvolvimento, o apoio e a promoção de programas } \\
\text { que conduzam saúde, em especial a saúde bucal, } \\
\text { tornando-se o local mais indicado para construção } \\
\text { desses hábitos saudáveis, quando inseridos em um } \\
\text { programa cotidiano-didático. }\end{array}$ \\
\hline 2018 & $\begin{array}{l}\text { OLIVEIRA, E.L; } \\
\text { RIATTO, S. G; VIEIRA, } \\
\text { A.P.S.B; CARVALHO, } \\
\text { G; FONSECA, M; } \\
\text { GUEDES, V; PEREIRA, } \\
\text { J; ROCHA, K }\end{array}$ & $\begin{array}{l}\text { A importância do } \\
\text { nível de } \\
\text { conhecimento dos } \\
\text { professores de escola } \\
\text { pública do ensino } \\
\text { fundamental sobre } \\
\text { saúde bucal - revisão } \\
\text { de literatura. }\end{array}$ & MEDLINE & $\begin{array}{l}\text { Os métodos de educação e motivação tem a finalidade } \\
\text { de esclarecer os pacientes sobre as doenças bucais e } \\
\text { mudar seus hábitos de higiene. O objetivo deste } \\
\text { trabalho foi o de descrever um programa de educação e } \\
\text { motivação para crianças em relação a saúde bucal em } \\
\text { escolas. }\end{array}$ \\
\hline 2019 & $\begin{array}{l}\text { CARDOSO; A, T, T. } \\
\text { CORREIA; E, P, A, S; } \\
\text { FERNANDES; D, E, G; } \\
\text { REZENDE; M, C, R, A }\end{array}$ & $\begin{array}{l}\text { Experiência de } \\
\text { educação em saúde } \\
\text { bucal em escola de } \\
\text { educação infantil na } \\
\text { república de cabo } \\
\text { verde, áfrica. }\end{array}$ & MEDLINE & $\begin{array}{l}\text { Dados do Observatório de Saúde Africana (African } \\
\text { Health Observatory), ligado à Organização Mundial de } \\
\text { Saúde (World Health Organization) apontam elevada } \\
\text { prevalência da cárie dentária, principalmente entre a } \\
\text { população escolar de Cabo Verde, elencando a } \\
\text { necessidade da priorização da promoção da saúde bucal } \\
\text { e da prevenção das doenças bucais pela integração da } \\
\text { saúde oral em outros programas tais como a formação } \\
\text { de professores, educadores de infância e outros } \\
\text { programas de responsabilidade na promoção de saúde. }\end{array}$ \\
\hline
\end{tabular}

Fonte: Dados de Pesquisa (2021). 
O Autor Valarelli (2011), à educação em saúde bucal é realizado por meio de palestras sistemáticas voltadas aos alunos de acordo com a faixa etária, aos professores e aos pais de alunos. No início de cada ano, realiza-se um levantamento epidemiológico, para identificação do quadro de saúde bucal das crianças e definição das estratégias e ações a serem implementadas. Nas palestras, são discutidos e praticados conteúdos referentes à escovação dentária, uso do fio dental, creme dental adequado para cada faixa etária, aspectos gerais da erupção dos dentes, aleitamento materno, dieta não cariogênica, características e controle do biofilme dentário, uso inteligente do açúcar, uso e indicação adequada do flúor, respiração bucal, a importância dos dentes, cuidados com a saúde bucal desde o nascimento e a responsabilidade dos pais no cuidado e na manutenção da saúde bucal dos filhos.

Avaliar o desenvolvimento de programas de educação e a motivação para saúde bucal em escolas são indispensáveis para a busca da melhoria da qualidade da saúde bucal, consequentemente havendo a redução de doenças bucais assim, o ambiente que acerca as crianças moldando atitudes que são fundamentais diante da vida das mesmas.

De acordo com Castro (2012) os recursos educativos que foram utilizados na pesquisa abordagens dos programas foram: modelos e macro modelos, folhetos explicativos, palestras e recursos audiovisuais, pôsteres, banners, vídeos livros e revistas específicos. Também foram utilizados recursos mais abrangentes, como: discussão e/ou discussão e reescrita de leitura auto instrutiva, fantoches, álbuns seriados, palestras com pais e responsáveis, aplicação de questionários, uso de robôs, sorriso e peças teatrais, jogos pedagógicos (gincana e competição), oficina pedagógica, atividades lúdicas.

Santos (2012), exatamente na infância que o procedimento das ações educativas deve ser efetivado devido á fase de crescimento e desenvolvimento tanto físico quanto intelectualmente, na infância que as crianças aprendem e vão tomando os cuidados e prevenção e ao longo de períodos com o conhecimento exposto as crianças internalizarão esses conhecimentos e levarão consigo esses estímulos de cuidados bucal ao longo da sua vida.

O papel da escola como parceira eficiente na formação de uma cultura de prevenção de doenças bucais, na medida em que atua como importante agente que transmiti as informações sobre saúde bucal, controle de açúcar na dieta e hábitos necessários para higiene da boca.

Garbin (2016), existem evidências de que a motivação e a educação em saúde são poderosas ferramentas quando empregadas de maneira agradável, atrativa e eficaz para transmissão de informações, sendo este o objetivo da promoção de saúde nas escolas. Nesse sentido, a literatura enfatiza que a escola é um ambiente propício para a aplicação de programas de educação em saúde por estar inserida em todas as dimensões do aprendizado.

Ações educativas também são realizadas com pais e professores. Essas ações acontecem em forma de diálogo e o assunto explorado depende das dúvidas e questionamentos que eles expõem nos encontros. Além disso, são realizadas reuniões mensais entre os acadêmicos, docentes e pós-graduandos para planejar as ações, discutir as principais dificuldades e compartilhar o aprendizado obtido, objetivando otimizar o programa.

Pereira (2017), existem evidências de que a motivação e a educação em saúde são poderosas ferramentas quando empregadas de maneira agradável, atrativa e eficaz para transmissão de informações, sendo este o objetivo da promoção de saúde nas escolas. Nesse sentido, a literatura enfatiza que a escola é um ambiente propício para a aplicação de programas de educação em saúde por estar inserida em todas as dimensões do aprendizado.

O processo é permanentemente monitorado e avaliado, utilizando-se alguns indicadores especialmente desenvolvidos para a ação. Até mesmo as diretoras e as professoras das escolas participam do processo de avaliação, contribuindo com sugestões e críticas.

Oliveira (2018), a odontologia pode, através do desenvolvimento de programas educativos, conscientizar e motivar, capacitando um número cada vez maior de indivíduos para atuar na pré-escola, construindo valores que os levem a agir, no seu dia a dia, em benefício de sua própria saúde e da saúde dos outros. No entanto, para que um programa educativo obtenha êxito, 
é necessário, ainda, o conhecimento da população alvo do programa. A falta de conhecimento dessas questões leva à implantação, muitas vezes inadequada, de políticas de saúde que não alcançam os resultados esperados e, em muitos casos, à impossibilidade de se saber se campanhas educativas podem vir a trazer algum benefício a determinada comunidade.

As crianças, a educação em saúde bucal se mostra altamente eficiente na diminuição do risco à cárie dentária, a qual pode evoluir rapidamente nesta faixa etária, comprometendo a qualidade de vida da criança e de seus familiares. Ademais, a escola de educação infantil se mostra como local privilegiado para o desenvolvimento de programas de educação e prevenção em saúde bucal, uma vez que reúne crianças com e sem acesso aos cuidados odontológicos privados.

Então a orientação em saúde bucal deve ser instituída já nos primeiros anos de vida, pois é nessa fase que a criança aprende facilmente e age de forma receptiva. As informações sobre saúde bucal associadas às ações preventivas, como escovação supervisionada e aplicação tópica de flúor, são eficazes na redução da experiência de cárie, sangramento gengival e acúmulo de biofilme. A associação dessas ações consolida os saberes aprendidos e age sobre os fatores responsáveis pelas doenças bucais (Garbin et al., 2016).

\section{Conclusão}

Diante da proposta da temática do artigo acadêmico, o ambiente que cerca as crianças vêm moldar as suas atitudes fundamentais diante da saúde e da vida delas. As suas atitudes e seus hábitos adotados perante as primeiras fases de vida serão carregados para as fases seguintes, quando se começa a assumir suas responsabilidades pelos próprios atos.

A educação e motivação a serem estabelecidas na escola tem grande impacto sobre o desempenho da criança com seus hábitos de saúde bucal. Esta pesquisa retrata as inovações nas atividades propostas no programa de educação em saúde bucal nas escolas. Estas inovações dependem das habilidades dos profissionais de saúde bucal, na implementação da educação em saúde, visando o desenvolvimento de programas de educação e motivação em saúde bucal em escolas.

Concluímos que os programas de saúde bucal nas escolas devem ser instituídos em todas as escolas para que haja uma saúde bucal de melhor qualidade para toda a população.

\section{Referências}

Antonio, L. P., Gouvêa, G. R., Souza, L. Z., \& Cortellazzi, K. L. Avaliação de diferentes métodos educativos em saúde bucal em crianças na faixa etária de 7 a 10 anos de idade. RFO. Passo Fundo, 20, 52-58.

Bulgareli, J., Cortellazzi, K. L., Ambrosano, G. M. B., Meneghim, M. C., De Faria, E. T., Mialhe, F. L., \& Pereira, A. C. A resolutividade em saúde bucal na atenção básica como instrumento para avaliação dos modelos de atenção, Ciênc. Saúde coletiva, 19(2).

Castro, C. O. Oliveira. K. S. Carvalho. R. B. Garbin. C. A. S. Santos. K.T. Programas de educação e prevenção em saúde bucal nas escolas: análise crítica de publicações nacionais. http://revodonto.bvsalud.org/scielo.php?script=sci_arttext\&pid=S1677-38882012000100009>.

Garbin. C. A. S. Soares. G. B. Martin. I. M. Garbin. A. J. I. Arcieri. M. A. Saúde bucal na escola: avaliação do conhecimento dos pais e da condição de saúde bucal das crianças. $R F O, 21,81-89$, http://revodonto.bvsalud.org/scielo.php?pid=S1413-40122016000100013\&script=sci_arttext>.

Leite, C. T. et al. Prática de Educação em Saúde percebida por escolares. CogitareEnferm. Ed.19. 1, 13-19.

Oliveira, E.L, Riatto, S. G, Vieira, A.P.S.B, Carvalho, G, Fonseca, M, Guedes, V, Pereira, J, Rocha, K. A Importância Do Nível De Conhecimento Dos Professores De Escola Pública Do Ensino Fundamental Sobre Saúde Bucal - Revisão De Literatura. Revista campo do saber https://periodicos.iesp.edu.br/index.php/campodosaber/article/view/165/142>.

Pereira, G. S., Carneiro, S. V., Martins, L. F. B., Bento, A. K. M., Leite, A. C. R. M., Silva, C. H. F. A Promoção Da Saúde Bucal No Contexto Escolar: Uma Revisão Integrativa. http://publicacoesacademicas.unicatolicaquixada.edu.br/index.php/recsaude/article/view/2111.

Santos, K. T., Garbin, A. J. I., Garbin, C. A. S. Saúde bucal nas escolas: relato de experiência. Rev. Ciênc. Ext. 8, 161-169. https://repositorio.unesp.br/bitstream/handle/11449/133364/ISSN1679-4605-2012-08-01-161-169.pdf?sequence=1>.

Valarelli, F. P., Franco, Valarelli, F. P., Franco, R. M., Sampaio, C. C., Mauad, C., Passos, L. L., Vitor, L. L. R. V., Machado, M. A. A. M., \& Oliveira, T. M. Importância dos programas de educação e motivação para saúde bucal em escolas: relato de experiência. Odontol. Clín. -Cient., 10 (2) 173-176, https://periodicos.iesp.edu.br/index.php/campodosaber/article/view/165>. 